\title{
DEVELOPMENT THE ELECTRONIC SYSTEM OF CONTINUES MODULAR SNAKE-LIKE-ROBOT
}

\author{
DANIEL ARMANDO GÓMEZ, JAVIER CAMILO TORRES VERA \\ El Bosque University, Electronics Engineering Department \\ Av. Cra 9 No. 131 A-02 Bogota, Colombia \\ HERNANDO LEÓN-RODRÍGUEZ \\ El Bosque University, Electronics Engineering and Bioengineering Department \\ Av. Cra 9 No. 131 A-02 Bogota, Colombia
}

\begin{abstract}
This project consists of the development of an electronic system to manipulate a snake like robot in a modular way. The electronic cards were implemented in a master-slave relationship for joint control of each mechanical module. These cards are composed of a DSPic30F4011, microchip 16-bit microcontroller that incorporates the CAN module, essential protocol for communication between cards, PWM outputs for motor control, analogue and digital ports; as well as a socket to connect to an external device through the UART. The firmware has been written in MikroC Pro. Each microcontroller implements the characteristic equation from the Hirose curves to generate a serpentine movement. These moves were simulated using ROS (Robotic Operating System in Rviz).
\end{abstract}

\section{Introduction}

The structural design of a snake is based on the repetition of its spine along its entire body, where only 3 types of bones make it up: the skull, the vertebrae and the ribs. The vertebral column is composed of between 100 and 400 vertebrae and each vertebra allows small movements in vertical and lateral direction, but the composition of so many vertebrae allows the snake a great flexibility and curvature with dramatically large forces.

The anatomy of the snake is composed of the same type of union and structure where each vertebra allows a rotation in the horizontal plane of 10-20 degrees and a rotation between 2-3 degrees in the vertical plane. [1] The locomotion system of the snake is very stable and the body is in constant contact with the ground at different points, allowing a low centre of mass and great traction which is easy to perceive its great ability with low energy consumption.

Snake-inspired robots were introduced in the 1970s by Shigeo Hirose. [2] Since then, several numbers of bio-inspired designs about snake like robots have been conceived and constructed. Although, the numerous designs of robots follow the kinematics and locomotion imitating the snake, they can change enormously in their physical configuration and purpose. For example, some robots are redundant; others are hyper-redundant while others may not have redundancy at all. [3] The first designs of snake-robots used traction wheels or tracks, while at present they can use passive wheels or without wheels at all. [4] Some designs are amphibious and can move effortlessly between terrestrial environments and water. [1] [6] [8] However, the demand for new types of robots is still present for rescue and inspection applications, where they do not 
require a robot capable of negotiating such conditions and difficulties in sewer lines, water networks and swamps. Robots based on thin and flexible snakes meet some of these needs. [5][7][9][15]

Commercially, robots for exploration of pipes are of many kinds, where each one of them fulfils different functions, mainly visual inspection of pipelines of drinking water and hydro-sanitary lines through video capture. [16][17][18] However, these robots have a very large disadvantage, the most of them require a very expensive and heavy transport logistics and for inspection services where the cost of using this equipment makes its frequent application difficult.

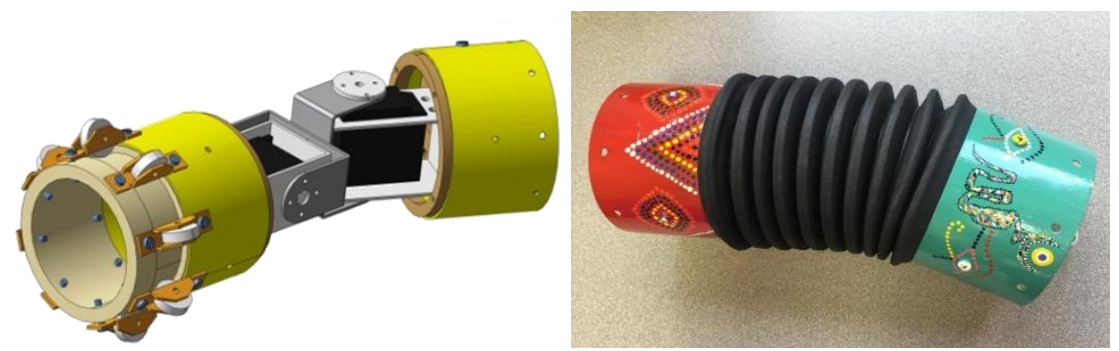

Figure 1: Conceptual design and module of snake like robot.

Figure 1 shows the conceptual design of the modular snake scalable robot; it is composed by 7 modules each one adapted by 2 degrees of freedom, generated by two servomotors. In this sense, the electronic design is focused in a modular way so that each mechanical joint carries its electronic backup and battery.

\section{Serpentine Locomotion}

There are different types of locomotion in snakes based on the condition of the terrain and the type of environment. In the development of the project the cards and control can reproduce any of the 4 types of snake locomotion; however, the applications of the control system and the communications of the cards focused the serpentine locomotion, as verification of control system. [10]

\section{Design of the electronic system}

The conditions of the electronic design are based on the components necessary to generate the movement of the robot based on its locomotion, environment, size, current and application. 


\subsection{Power requirement and voltage regulation}

The selected batteries are based on the power delivered, charging time and the space gap within of the modules. The selected batteries are lithium-polymer $72 \times 34 \times 14 \mathrm{~mm} 1000 \mathrm{~mA}$, composed of two cells of 7.4 volts.

Due to the high efficiency of buck step-down type regulators, table 1 is showing the characteristics of the following devices that have been selected to satisfy current requirements of approximately 2.5 Amps, efficiency of $85 \%$ to $95 \%$, capable to inverse voltage, and smaller as possible.

\subsection{Microcontroller and communication protocol}

The microcontroller that has been chosen mainly based on its 16-bit architecture; additionally, it has CAN communication protocol incorporated as a final purpose of control and position of the servomotors. Its parameters are: architecture:16-bits, CPU speed 30 MPS, type of minority: flash, memory: 48 $\mathrm{KB}$, ram: $2 \mathrm{~KB}$, temperature range 40 to $125 \mathrm{C}$, operating voltage 2.5 to $5.5 \mathrm{~V}$, Number of I/O ports: 40, digital communication peripherals: 2-UART, 1-SPI; 1I2C, analogue peripherals: 1-A/D, 9x10-bits; $1000 \mathrm{kps,} \mathrm{communication}$ protocol: CAN, PWM channels: 6 and parallel port: GPIO.

The proposed network protocol for internal communication between the electronic systems is a master-slave connexion. At the beginner, was considered to be performed through RS485, however, the CAN Bus was chosen, used in the automotive industry due to its robust protocol, which corrects transmission errors and It is invulnerable to electromagnetic disturbances, thanks to its physical layer requirements that are a shielded in a differential pair.

\section{Mathematical analysis}

Based on the cinematic analysis of Professor Hirose; [2] the behaviour and locomotion of a snake expresses the serpenoid curves and their joint trajectories as follow: [1] [2] [10] [14]

\subsection{Serpenoidal Curves -Hirose}

The following equations express the serpenoid curves proposed by Hirose. The length of the segment along the serpent is represented; $a, b$ and $c$ are parameters that determine the shape of the curve.

$$
\begin{aligned}
& X(s)=\int_{0}^{s} \cos (a \cos b \sigma+c \sigma) d \sigma \\
& Y(s)=\int_{0}^{s} \sin (a \cos b \sigma+c \sigma) d \sigma
\end{aligned}
$$




\subsection{Articular trajectories}

The joint trajectories determine the angles that the joints must develop over time to generate a serpenoid curve; these equations are those which are executed by the microcontrollers on each electronic card in order to control the angles of the servomotors. The equation (3) contains a new component $\omega$, which is determined by $2 \pi \mathrm{f}$, where $f$ is the frequency with the curve generated by equations (1) and (2).

$$
\emptyset_{i}(t)=2 \alpha \sin (\omega t+(i-1) \beta)+\gamma
$$

Where: (4), (5) and (6) come from the same parameters $a, b$ and $c$ of the serpenoid curve, [11]

$$
\begin{gathered}
\beta=\frac{b}{n} \\
\gamma=\frac{-c}{n} \\
\alpha=2 a\left|\sin \left(\frac{\beta}{2}\right)\right|
\end{gathered}
$$

As a result: $i$, is the number of the joint, that is, the first joint will have an equation with $i=1$, the second with $i=2$ and so on.

\section{Analysis and results}

\subsection{Electronics card}

The figure 2 showing the electronic card implemented in each mechanical module of the robot structure. This card execute the master or slave control condition; the result is been done with a single card design allows to be configured as a master or slave according to the needs. Each card is identified with an internal code that permits to know its location on the robotic structure.
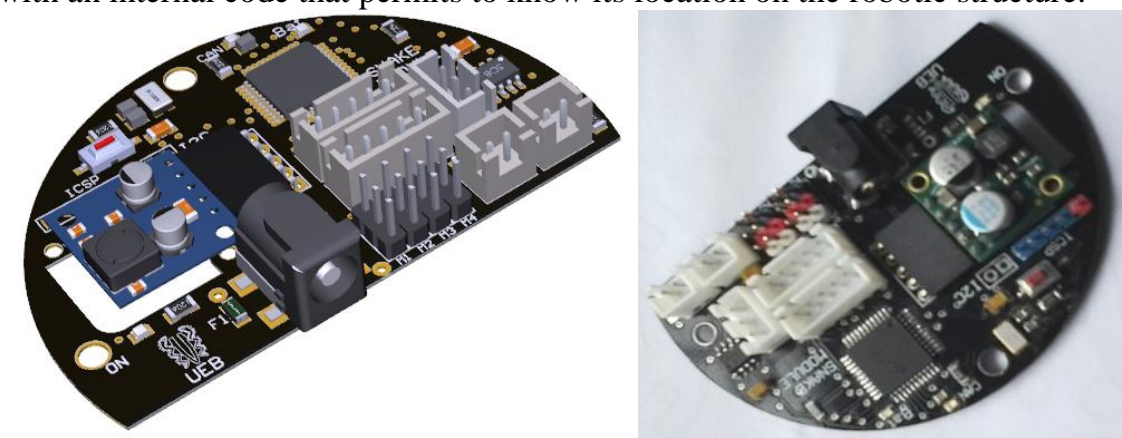

Figure 2: photorealistic image and picture of the developed control card.

The control and communication system of the developed card is presented in figure 3; this shows the diagram of any device with $5 \mathrm{~V}$ supply voltage and logic, such as RF, Bluetooth, wifi modules that support the TTL/UART 
interface. The diagram also showing the following the communication protocols: CAN; Uart, I2C that allow controlling any device such as sensors, servomotors, LEDs, etc. all them that support at the same time by the interface.

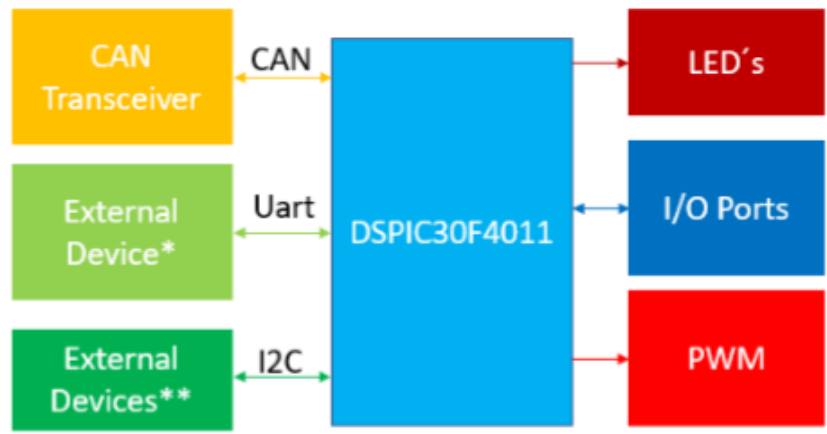

Figure 3: Block diagram of the electronic card.

\subsection{Mathematical analysis}

Figure 4-left is showing multiple serpenoid curves generated by the modification of different parameters in control, such as: frequency, amplitude and phase shifting.
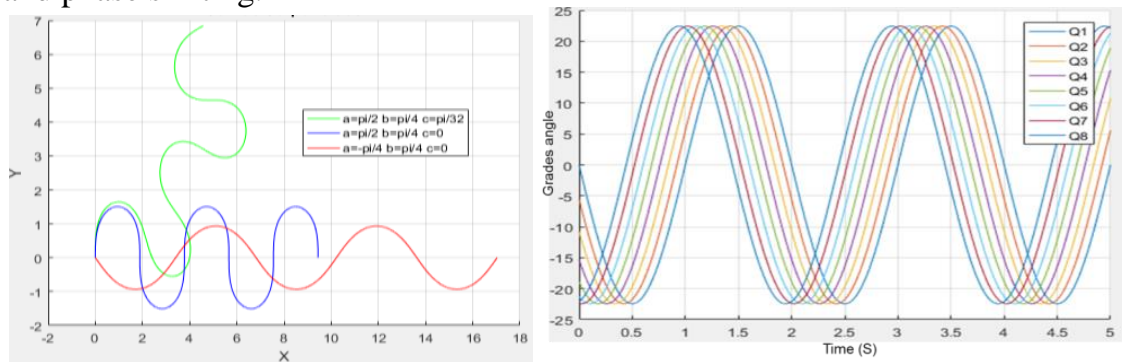

Figure 4: left: Simulation of trajectory behaviour and locomotion of the modular snake-robot; right: Articular angles with respect to time: left: a=-pi/4, c=pi/2, w=2pi*(0.5).

Figures 4-right is showing the simulations of each joint of the robotic system covering the joint angles (Q1 to Q8) with respect to time with different parameters $a, b$ and $c$, based on equations 1 and 2 .

Figure 4-right also showing the path of each of the joint modules to complete the serpenoid curve; it should be notice that if these graphs were developed with $n=7$, (number of joints) the results are similar.

\section{Simulation of robot kinematics}

The simulation includes the implementation of the joint trajectories in the mechanical modules of the robot. As a result, the robot design has been taken to 
the URDF format compatible by the RVIZ simulator and through the publisher and subscriber of ROS. The described angles previously and the equations produce the simulated trajectories on RVIZ. [12] [13]

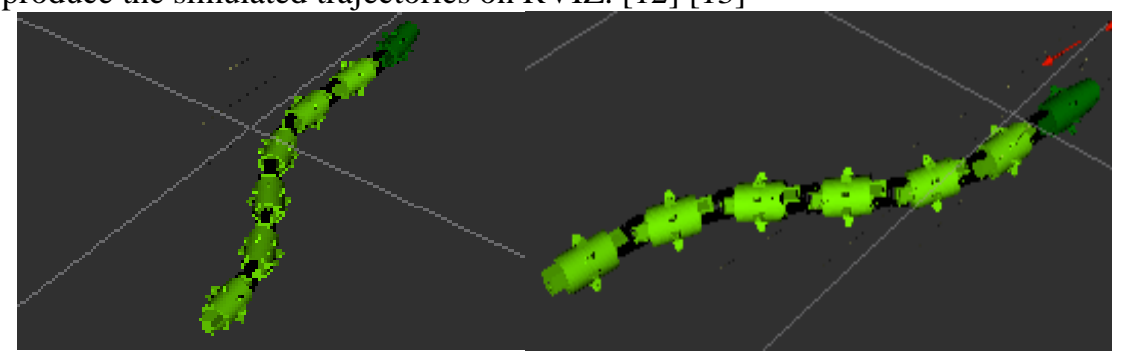

Figure 5: Result of robot simulation developing serpenoid curve in RVIZ-ROS.

The figure 5 is showing the executed simulation in RVIZ, in addition is validating that the robot could move in a serpentine way. However, in this first implementation, aspects such as the weight of the robot, friction and floor uniformity were not considered.

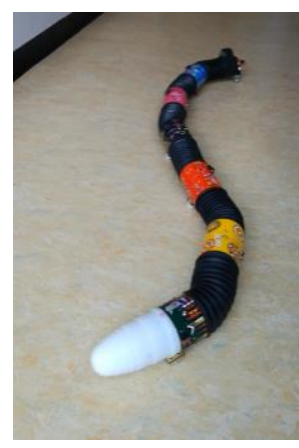

$a$

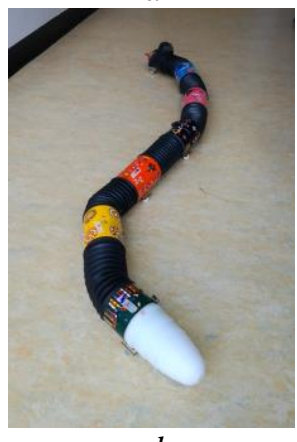

$d$

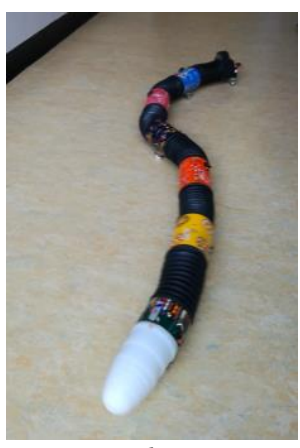

$b$

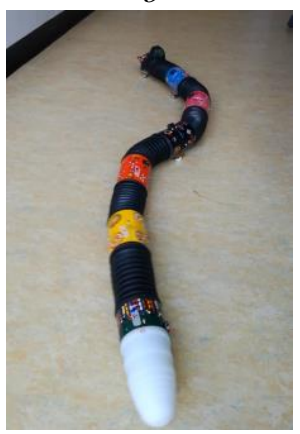

$e$

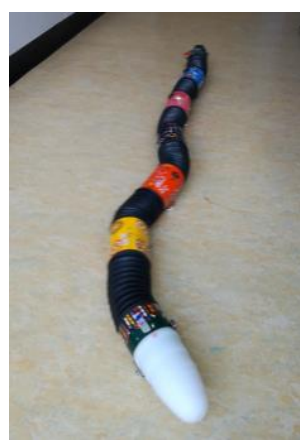

$c$

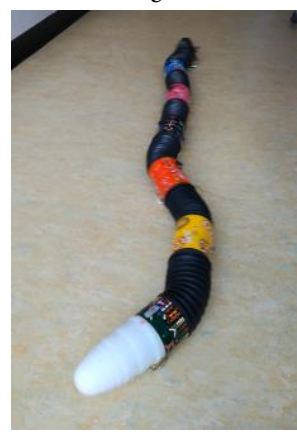

$f$

Figure 6: Motion sequence of the implemented robot like snake.

Figures 6 are showing the final implementation of the locomotion by the snake like robot based on the serpentine movements. Further research and word need 
to be done in other to improve and produce soft-motion and better performance. Nevertheless, the control cards with CAN communications were well executed by the controllers, which were the aim of this preliminary research.

\section{Conclusions}

This project presents in a superficial way the design criteria for a particular electronic system to control a robot, these must cover its processing unit such as the microcontroller, its power supply and regulation, the peripherals that must be contemplated, either that you want to use sensors. Follow the rules as those established in IPC-2221 to develop PCBs with high quality standards.

The project presents the importance of a mathematical analysis in terms of robotics and its respective simulation to check its effectiveness. However, there is a huge gap between the simulation and the real, since some physical variables did not contemplated precisely.

\section{Acknowledgment}

This project openly thanks the contributions for the development of the control cards by the researchers: Michael Canu, Cecilia Murrugara; and especially to the Researcher Juan David Hernández of the University of Girona for his valuable contribution and knowledge in the management and control of ROS.

\section{References}

1. J. K. Hopkins, B.W. Spranklin, and S.K. Gupta, A survey of snake-inspired robot designs. Bioinspiration and Biomimetics, 4(2):021001, 2009.

2. Shigeo Hirose and Hiroya Yamada, Snake-Like Robots, Machine Design of Biologically Inspired Robots, IEEE Robotics \& Automation Magazine, March 2009

3. Kevin J. Dowling, Limbless Locomotion: Learning to Crawl with a Snake Robot, The Robotics Institute Carnegie Mellon University and NASA Graduate Fellowships, December 1997.

4. Saori Sugita, Kazunori Ogami, Guarnieri Michele, Shigeo Hirose, and Kensuke Takita, A Study on the Mechanism and Locomotion Strategy for New Snake-Like Robot Active Cord Mechanism-Slime model 1 ACM-S1, Journal of Robotics andMechatronicsVol.20 No.2, 2008.

5. Cornell Wright, Austin Buchan, Ben Brown, Jason Geist, Michael Schwerin, David Rollinson, Matthew Tesch, and Howie Choset, Design and Architecture of the Unified Modular Snake Robot, 2012 IEEE International Conference. 
6. Shumei Yu, Shugen Ma, Bin Li, Yuechao Wang, An Amphibious Snakelike Robot: Design and Motion Experiments on Ground and in Water, Proceedings of the 2009 IEEE International Conference on Information and Automation, June 22 -25, 2009, Zhuhai/Macau, China.

7. Aksel A. Transeth, Remco I. Leine, Christoph Glocker and Kristin Y. Pettersen, 3D Snake Robot Motion: Nonsmooth Modeling, Simulations, and Experiments, IEEE transactions on robotics, vol. 24, no. 2, April 2008.

8. Hiroya Yamada and Shigeo Hirose, Study of a 2-DOF Joint for the Small Active Cord Mechanism, 2009 IEEE International Conference on Robotics and Automation Kobe International Conference Center, Kobe, Japan, May 12-17, 2009.

9. A.J. Ijspeert and A. Crespi; Online trajectory generation in an amphibious snake robot using a lamprey-like central pattern generator model, Proceedings of the 2007 IEEE International Conference on Robotics and Automation (ICRA 2007), pages 262-268,

10. David Rollinson; Control and Design of Snake Robots; School of Computer Science Carnegie Mellon University, 2014.

11. Chaohui Gong, Matthew J. Travers, Henry C. Astley, Lu Li, Joseph R. Mendelson, Daniel I. Goldman and Howie Choset; Kinematic gait synthesis for snake robots; The International Journal of Robotics Research 1-14, DOI: 10.1177/0278364915593793, 2015

12. Filippo Sanfilippo, Øyvind Stavdahl and Pal Liljeback; SnakeSIM: A Snake Robot Simulation Framework for Perception-Driven Obstacle-Aided Locomotion; Proceeding of the 2nd International Symposium on Swarm Behavior and Bio-Inspired Robotics (SWARM), Kyoto, Japan, 2017.

13. Filippo Sanfilippo, Øyvind Stavdahl and Pal Liljeback; SnakeSIM: a ROSbased Rapid-Prototyping Framework for Perception-Driven ObstacleAided Locomotion of Snake Robots; Proceeding of the IEEE International Conference on Robotics and Biomimetics (ROBIO 2017).

14. Stian Grøttum Danielsen; Perception-Driven Obstacle-Aided Locomotion for snake robots, linking virtual to real prototypes; Norwegian University of Science and Technology, 2017

15. Biorobotics laboratory; (2018, May 16). Retrieved from: http://biorobotics.ri.cmu.edu/projects/modsnake/

16. Rausch Electronics USA; (2018, May 20). Retrieved from: http://rauschusa.com/

17. Aries Industries; (2018, June 13). Retrieved from: http://www.ariesindustries.com/

18. Ibak; (2018, June 10). Retrieved from: http://www.ibak.de 